\title{
Viewpoint
}

\section{Evidence based diagnostic microbiology: has its time come?}

The concept of evidence based practice (EBP) has evolved in response to technological advances and external pressure to increase efficiency and to contain the cost of health care. ${ }^{1}$ The ambitious aim of evidence based health care, and evidence based pathology in particular, is to advance clinical diagnosis and prognosis of disease through research and dissemination of new knowledge that meets high standards of critical review. ${ }^{2}$ EBP requirements and principles have been reviewed elsewhere. ${ }^{24}$ Importantly, the approaches to evidence based treatment are well established; however, evidence based diagnosis is much less developed. The purpose of this commentary is to outline the current status of EBP in diagnostic microbiology and to suggest some steps that pathologists can take to promote patient outcome oriented microbiology.

\section{Emerging diagnostic challenges}

Pathologists have been overwhelmed in recent years by the seemingly relentless growth of requirements to improve the effectiveness and efficiency of laboratory practice. In addition, the diagnostic microbiology market is becoming increasingly sensitive to costs and customer requirements. Cost cutting has a considerable negative effect on microbiology research and development. Adding fuel to this concern, there is an emerging view that pathology is a clinical service that has to adjust to the expectations of patients and health service providers. The focus of quality control has been shifting from zero defects to zero defections of referring physicians.

Diagnostic microbiology services are increasingly criticised for encouraging higher rates of testing without concomitant benefits. At the same time, in a recent systematic review of laboratory audits, $46 \%$ of microbiology diagnostic tests were found to be inappropriate. ${ }^{5}$ How often appropriate tests are not requested has not been studied systematically. ${ }^{56}$

The role of the microbiology laboratory in the diagnosis of mild infections (acute bronchitis, urinary tract infections, community acquired pneumonia, etc) has been challenged. Even for seriously ill patients, controversy exists over the diagnostic value of many specimens obtained routinely. ${ }^{7}$ We are still unsure of what constitute acceptable rates of diagnostic failure or laboratory errors. We often culture swabs of-for example, perianal abscesses or decubitus ulcers - with a clear understanding that to provide a result of doubtful clinical value consumes substantial laboratory resources.

The increasing use of rapid diagnostic assays that replace traditional microbiological cultures can affect surveillance, prescribing, and medicolegal practice. For example, presumptive but quick diagnosis based on $\operatorname{IgM}$ antibody detection has largely replaced conventional assessment of antibody titres in paired sera. The use of more economical near patient testing methods redefines the location and delivery model for laboratory medicine and shifts the traditional base of diagnostic testing from laboratories to the community. ${ }^{8}$
Is the art of clinical microbiology evidence based?

The key principle of EBP is that different levels of evidence lead to stronger inferences than others. ${ }^{9}$ Well conducted, integrative studies that combine the results of randomised trials (such as meta-analyses) and large individual randomised control trials (RCTs) provide the most persuasive evidence. Observational and exploratory studies defining changes in cases and controls and developing technical methods rank lowest on the hierarchy because clinical experience is highly open to bias. ${ }^{10}$ However, information for most laboratory tests is only available in the form of case control studies. There are only a few effectiveness studies looking at the impact of laboratory diagnoses on clinical outcomes and on efficiency of the diagnostic process. The number of RCTs and systematic reviews applicable to the practice of clinical microbiology also remains limited. ${ }^{6}{ }^{8}$ In the recent edition of the Cochrane infectious diseases group review (Cochrane Library, issue 4 for 1999), we identified 34 systematic reviews of the use of vaccines for prevention or antibiotics for treatment of infection, but none on laboratory diagnosis of infective causes.

EBP encourages us to focus on the ability of a test to change our minds from what we thought before the test (pre-test probability of infection) to what we think afterwards (post-test probability). Diagnostic tests that produce significant change from pre-test to post-test probabilities are likely to be most useful. ${ }^{11}$ However, the number of studies and clinical situations in which pre-test and post-test probabilities of infection have been rigorously determined is relatively small. ${ }^{6}{ }^{12}$ Clearly, many studies are performed to assess, or even to promote, the role of a specific kit or system, rather than to investigate the laboratory diagnosis of a clinical entity. ${ }^{8}$

New ideas around likelihood ratios have expanded the old fashioned concepts of sensitivity and specificity. For example, likelihood ratios of $>10$, calculated to estimate the post-test probability of the outcome, significantly increase the probability that infection is present. To achieve these ratios population size should exceed 500 patients in the disease and non-disease branches, making this target difficult to achieve. Thus, only a minority of published studies (less that one third in one systematic review ${ }^{13}$ ) report data in a way that allows likelihood ratios to be calculated. Those that do so often report ratios that lie within this indeterminate range (between 0.1 and 10) and thus they appear to be of limited value for predicting the presence or absence of disease.

In brief, we need more and better evidence than is currently available for most of the decisions made in the microbiology laboratory about test selection and result interpretation. Despite a commitment to a quality diagnostic service, the practice of evidence based medicine remains a challenge for microbiologists. Furthermore, diagnostic microbiology relies heavily on subjective, expert opinion for microbial identification and result interpretation, which leads to problems with standardisation and reporting. 
Table 1 Comparison of outcome oriented and experience based practice in microbiology

\begin{tabular}{ll}
\hline Outcome oriented practice & Experience based practice \\
\hline Focus & \\
- Rapid, simple methods & - Conventional techniques \\
- Presumptive descriptive results & - Complete investigation \\
- Clinically relevant reporting & - "State of art" scientific reporting \\
- Better patient outcome at lower cost & - Laboratory output \\
$\begin{array}{l}\text { - Competitive service and utilisation } \\
\text { control }\end{array}$ & - Academic excellence \\
$\begin{array}{l}\text { Concept } \\
\text { - "Duty of cure" }\end{array}$ & - "Duty of care" \\
- Relicit, can be evaluated & - Implicit, usually cannot be measured \\
diagnostic pathways & - Focused on a specific patient \\
\hline
\end{tabular}

\section{Can evidence based pathology provide better} medical care?

There is no convincing answer to this question yet. Nevertheless, it is likely to be "yes" if we believe that microbiological results contribute greatly to patient management and public health. ${ }^{14}$ International standards require laboratories to implement and maintain quality systems to ensure uniform diagnostic processes throughout the entire scope of laboratory activities. To meet these challenges, cooperative efforts and the use of technological advances are warranted. Clearly, few clinical questions are studied by a single group only. Thus, the collaborative, interdisciplinary, multicentre trial model pioneered by human immunodeficiency virus research ${ }^{14}$ could provide a model to deal with the challenges of EBP.

A reassessment of laboratory performance evaluation methods is needed. Simply counting the number of infections diagnosed is of limited value. Diagnostic microbiology is expected to provide short and longterm clinical outcome measures, both for reactive, symptom driven, and proactive, health promoting infection management strategies. In addition, we must define how much additional cost can be justified by marginal increases in health care benefit through reporting more information. Without this, laboratory utilisation may swing from overtesting to undertesting.

Evidence and knowledge is constantly being reexamined and the half life of the truth of evidence in infectious disease is limited. ${ }^{14}$ Advances in mathematical modelling techniques may save time and resources in the evaluation of diagnostic pathways, and their potential risks and benefits. Information technology can provide quick access to high quality research evidence at the patient's bedside or in the clinician's office. For example, electronic "trial banks" have been proposed to overcome the lack of a standardised structure for trial reports and problems with their retrieval and dissemination. ${ }^{15}$ According to this model, as part of the publication of the results, clinical trials can be reported into an electronic database, which is accessible to users for analysis. These databases may not only provide well defined data but allow the performance of prospective continuous meta-analyses and outcome studies to improve good practices and eliminate poor ones.

\section{Conclusion: harmonising EBP with clinical experience}

It is apparent that the most fundamental change needed, if microbiology is to make meaningful progress in EBP, is a cultural one. Pathologists have to harmonise traditional scientific experience with outcome oriented practice aimed at effective clinical interventions. These approaches often represent different concepts and performance assessment priorities (table 1).

The EBP movement is driven largely by a shift away from the traditional view of health care as a predominantly public service that is exempt from market forces, towards a view that it is also an economic entity. Nevertheless, it supports the view that competition in the pathology marketplace should be based not on the cost of service but rather on its quality. Our acceptance of the neologism "evidence based pathology" should not allow humanitarian considerations to be overshadowed by financial concerns and should not obscure the real goal of improving individual and public health outcomes.

There is an increasing pressure to move away from the traditional academic view that no stone should be left unturned in the investigation of the diseased individual to the cost effective use of health care resources. Nevertheless, clinical effectiveness but not cost effectiveness should remains the key concept of evidence based pathology. EBP may reduce the element of uncertainty in medical practice; however, it is unlikely to be a panacea for health care delivery. Combining the experience of clinicians and microbiologists with scientific evidence will help to overcome the problem of complexity, uncertainty, and imperfect information in diagnostic microbiology and to optimise the impact of laboratory results on patient management.

V SINTCHENKO

Centre for Health Informatics, University of New South Wales, Sydney 2052, Australia

\section{G L GILBERT}

Centre for Infectious Diseases and Microbiology Laboratory Services, Institute of Clinical Pathology and Medical Research, Westmead Hospital, Westmead 2145, New South Wales, Australia

vitalis@icpmr.wsahs.nsw.gov.au

1 Batstone G. Practising by the evidence: the role of pathology. 7 Clin Pathol 1997;50:447-8.

2 Centre for Evidence-Based Pathology, Queens Medical Centre, University of Nottingham (http//www.ccc.nottingham.ac.uk/ mpzjlowe/evcent.html). 3 Fleming KA. Evidence-based pathology. F Pathol 1996;179:127-28.

4 Grahame-Smith D. Evidence-based medicine: challenging the orthodoxy. $f$ $R$ Soc Med 1998;91(suppl 35):7-11.

5 Van Walraven C, Naylor CD. Do we know what inappropriate laboratory utilization is? A systematic review of laboratory clinical audits. $\mathcal{F} A M A$ 1998;280:550-8.

6 Moore RA. Current perspectives in evidence-based laboratory medicine. $\mathrm{Br}$ f Biomed Sci 1999;56:226-33.

7 Grossman RF. Evidence-based assessment of diagnostic tests for ventilatorassociated pneumonia. Chest 2000;117(suppl):S177-181.

8 Borriello SP. Near patient microbiological tests. BM7 1999;319:298-301.

9 Taubes G. Looking for the evidence in medicine. Science 1996;272:22-4.

10 Lijmer JG, Mol BW, Heisterkamp S, et al. Empirical evidence of design-related bias in studies of diagnostic tests. FAMA 1999;282:1061-6.

11 Goodman SN. Toward evidence-based medical statistics. 2: The Bayes factor. Ann Intern Med 1999;130:1005-13.

12 Reid MC, Lachs MS, Feinstein AR. Use of methodological standards in diagnostic test research: getting better but still not good. $7 A M A 1995 ; 274$ : 645-51.

13 Fowlie PW, Schmidt B. Diagnostic tests for bacterial infection from birth to 90 days - a systematic review. Arch Dis Child Fetal Neonatal Ed 1998; 78:F92-8.

14 Ioannidis JPA, Lau J. State of the evidence: current status and prospects of meta-analysis in infectious diseases. Clin Infect Dis 1999;29:1178-85.

15 Sim I, Owens DK, Lavori PW, et al. Electronic trial banks: a complimentary method for reporting randomised trials. Med Decis Making 2000:20:44050 . 